\title{
Avaliação do desempenho dos exames anatomopatológico e histopatológico na inspeção post mortem de bovinos suspeitos ou reagentes à prova de tuberculinização
}

\author{
Performance evaluation of the anatomopathological and \\ histopathological analysis in the post mortem inspection of bovine \\ suspects or reagents to the tuberculin test
}

\author{
Paulo Sérgio de Arruda Pinto, ${ }^{*}$ Marlene Isabel Vargas Viloria, ${ }^{*}$ José Eurico de Faria, ${ }^{*}$ Laerte Pereira de Almeida, ${ }^{* \star}$
}

\begin{abstract}
Resumo
A tuberculose é uma zoonose associada a sérios prejuízos sanitários e econômicos. A infecção por tuberculose bovina é tradicionalmente identificada pela conversão à tuberculina. Embora esteja previsto o emprego de exames laboratoriais no seu diagnóstico, o exame anatomopatológico tem sido o principal recurso utilizado na inspeção post mortem de animais em matadouros. $\mathrm{O}$ experimento foi conduzido com 50 bovinos reagentes ou suspeitos à prova de tuberculinização intradérmica ano-caudal, com confirmação da prova comparativa cervical. Todos estes animais foram submetidos ao exame anatomopatológico. Visando o exame histopatológico, foram coletados dos bovinos abatidos, sob inspeção, tecidos contendo lesões macroscópicas sugestivas (19) ou típicas (12) de tuberculose e de 19 outros linfonodos cervicais superficiais e ilíacos internos, aleatoriamente escolhidos para exame. Apenas $18 \%$ dos bovinos tuberculina positivos e nenhum dos suspeitos apresentaram lesões histológicas, sendo todas estas associadas com lesões anatomopatológicas típicas. Frente ao exame anatomopatológico, o histopatológico obteve uma baixa sensibilidade de $19 \%$, mas sua especificidade foi de $100 \%$, indicando ser um apreciável recurso auxiliar de diagnóstico da tuberculose em situações especiais, como a de abate de bovinos tuberculina positivos e suspeitos. O número de lesões encontradas por bovino tuberculina positivo foi de 1,53. Os locais destacados no diagnóstico da tuberculose bovina foram os tecidos respiratórios e digestivos, sobretudo os linfonodos medistínicos e bronquiais e o pulmão. O exame histopatológico apresentou menor sensibilidade, entretanto, maior especificidade que o anatomopatológico; porém, quando o exame anatomopatológico é realizado com método detalhado, este se apresenta como um teste mais sensível, comportando-se como um satisfatório exame em inspeção post mortem.
\end{abstract}

Palavras-chave: tuberculose, exame anatomopatológico, exame histopatológico, tuberculina, inspeção de carnes.

\begin{abstract}
The tuberculosis is a zoonosis associated to serious sanitary and economic losses. The bovine tuberculosis infection is traditionally identified by the tuberculin test. Although the employment of laboratory exams is commum in its diagnosis, the anatomopathological exam has been the main method used in the post-mortem inspection of tuberculosis in slaughterhouses. The experiment was carried out with 50 positives or suspects bovine to the intradermic tuberculin test, with the cervical comparative test confirmation. All these animals were submitted to the anatomopathological exam. For the histopatological exam, samples were collected of bovine tissues with suggestive (19) or typical (12) macroscopic lesions and other (19) superficial cervical and internal iliac linfonodes. Only $18 \%$ of the positive tuberculin bovine and none of the suspects was presented histological lesions, and all these was associated with typical anatomopathological lesions. Compared to the anatomopathological, the histopatological exam obtained a low sensitivity (19\%), but its specificity was excellent (100\%), showing to be an appreciable auxiliary method of tuberculosis diagnosis in special situations, as the one of tuberculin positive and suspected bovine. Was found the average number of lesions of 1,53 by positive tuberculin bovine. The best sites to the diagnosis of the bovine tuberculosis were the respiratory and digestive systems, mainly the lung and the mediastinics and bronchial linfonodes. The histopathological exam was presented as a slowest sensitivity and highest specificity that the anatomopathological. Nevertheless, the anatomopathological exam was showed as a satisfactory exam in post-mortem inspection, because of its sensitivity still most high, when the exam is accomplished with detailed method.
\end{abstract}

Keywords: tuberculosis, anatomopathological analysis, histopathological analysis, tuberculin, meat inspection.

\footnotetext{
* Departamento de Veterinária - Universidade Federal de Viçosa - CEP 36571-000 - Viçosa, MG. E-mail: pintopsa@ufv.br

** Faculdade de Medicina Veterinária - Universidade Federal de Uberlândia - Uberlândia, MG.
} 


\section{Introdução}

A tuberculose é uma zoonose associada a sérios prejuízos sanitários e econômicos.

Um terço da população mundial carece de programas de segurança agrícola e alimentar, permanecendo sob substancial risco de contrair a tuberculose por M. bovis (Nelson, 1999).

Entre 1985 e 1995, houve uma retomada do crescimento da mortalidade por tuberculose em São Paulo (4,08\% por ano), refletindo a crescente prevalência da co-infecção por Mycobacterium sp e por HIV (Antunes e Waldman, 1999).

Embora a carne proveniente de um animal com lesões localizadas ou tuberculina positivos ainda possa ser utilizada para consumo humano na forma de aproveitamento condicional por cocção, ela perde $50 \%$ do seu valor comercial, quando definida esta destinação (Roberts, 1986).

$O$ custo total de tratamento e perdas salariais de cada paciente com tuberculose por M. bovis nos Estados Unidos foi de US\$ 8.275,00, segundo cálculo de Roberts (1986). O custo anual do programa de erradicação da tuberculose na Austrália foi de cerca 60 milhões de dólares (Corner et al., 1990).

A infecção por tuberculose bovina é tradicionalmente identificada pela conversão à tuberculina.

Uma vez infectado, o bovino pode desenvolver uma lesão primária "fechada", que pode regredir ou progredir para "aberta" ou para locais distantes, ambas podendo também regredir ou progredir; ou seja, as lesões resolvidas podem se reativar no mesmo local ou em outro distante, caracterizando-se como uma doença crônica (Grange e Yates, 1994).

Embora esteja previsto o emprego de exames laboratoriais, o exame anatomopatológico tem sido o principal recurso utilizado na inspeção post mortem de animais em estabelecimentos de abate. Porém, a probabilidade de passarem lesões despercebidas à inspeção oficial de bovinos, mais comuns nas infecções discretas com lesões únicas, foi estimada em 47\%, no sistema australiano de inspeção (Corner et al., 1990).

O Regulamento de Inspeção Industrial e Sanitária de Produtos de Origem Animal - RIISPOA, do Serviço de Inspeção Federal (Lei no 1253, art. 119 e 196; http:Ilwww.agricultura. gov.brlsdaldipoa; Brasil, 1980) estabelece a decisão sanitária oficial sobre a tuberculose em matadouros, determinando critérios de rejeição parcial, aproveitamento condicional e condenação de carcaças, segundo sua condição anatomopatológica e ainda determina que os animais levados ao abate, para controle de provas de tuberculinização, sejam abatidos em separado, submetendo-o a uma inspeção mais rigorosa.

As lesões macroscópicas crônicas da tuberculose bovina caracterizam-se por nódulos granulomatosos típicos, chamados "tubérculos", semelhantes às lesões do linfossarcoma e de linfadenites inespecíficas, por coloração amarelada, presença de cápsula, aspecto caseoso e consistência calcárea e se localizam preferencialmente em linfonodos mediastínicos, bronquiais e mesentéricos, em função da porta de entrada do bacilo, aerógena ou digestiva (Reis et al., 1995; Reis et al.,1996).

Microscopicamente, as lesões tuberculosas clássicas aparecem no estágio crônico da doença nos bovinos e são mani- festadas por uma necrose de caseificação e, ou de calcificação central, circundada por macrófagos de núcleo grande e claro e abundante citoplasma, dispostos uns ao lado dos outros, chamados "células epitelióides", que se fundem formando as células gigantes multinucleadas (Souza et al., 1999).

Com freqüência variável, se encontram animais anérgicos, que apresentam lesões na inspeção postmortem; pelo contrário, são encontrados animais tuberculina positivos sem lesões, como ocorre em animal com infecção recente (CPZ, 1979). A permanência da hipersensibilidade à prova de tuberculinização em animais recuperados de uma infecção também ocorre, por isso atenção especial deve ser dispensada aos exames macroscópico e histológico de bovinos suspeitos ou reagentes a esta prova, por permitirem maior acurácia do diagnóstico (Corner et al., 1990).

Langenegger et al. (1976) evidenciaram em bovinos, o desenvolvimento de reações paralérgicas à prova de tuberculinização associadas principalmente a micobactérias do grupo $M$. intracellulare-scrofulaceum, sobretudo nas propriedades altamente contaminadas, que criam bovinos e suínos. Também é relatada a possibilidade de ocorrência de reação inespecífica em bovinos, provocada por $M$. intracellulare, quando se usa a tuberculina aviária em testes comparativos (Castro e Nemoto, 1972).

Os programas de tuberculinização dos rebanhos têm sido e se mantido como um dos recursos de rastreabilidade da tuberculose detectada em matadouros. O declínio das taxas de tuberculose bovina vem ocorrendo devido ao incremento duplo dos programas de tuberculinização e de inspeção da carne, resultando em redução do número de animais infectados no campo e no matadouro (Roberts, 1986). Entretanto, os benefícios do controle da tuberculose bovina para a saúde pública e animal depende de novas técnicas e procedimentos laboratoriais, sobretudo as de baixo custo (Roberts, 1986).

Dada a sua importância na prática de inspeção post mortem, há necessidade de se avaliar o exame anatomopatológico de bovinos, a partir de parâmetros mais objetivos, como a investigação histopatológica, para assegurar uma boa sanidade da carne inspecionada em matadouros e, conseqüentemente, a saúde pública (Benites et al., 1999).

Pretendeu-se com este trabalho avaliar o desempenho do exame histopatológico como recurso de apoio à inspeção post mortem em situação especial, quando os bovinos eram suspeitos ou reagentes ao teste de tuberculinização e apresentaram lesões macroscópicas.

\section{Metodologia}

O experimento foi conduzido com 50 bovinos em bom estado de saúde, porém reagentes ou suspeitos à prova de tuberculinização intradérmica ano-caudal, com confirmação da prova comparativa cervical. Todos estes animais foram submetidos ao exame anatomopatológico.

Visando o exame histopatológico, foram coletados dos bovinos abatidos, sob inspeção, tecidos contendo lesões macroscópicas sugestivas (19) ou típicas (12) de tuberculose e de 19 outros linfonodos cervicais superficiais e ilíacos internos, aleatoriamente escolhidos para exame. 
O método de exploração anatomopatológica dos tecidos anteriormente mencionados se baseou nas normas do exame post mortem do Serviço de Inspeção Federal (Brasil, 1971).

O material coletado foi fixado em formol $10 \%$ neutro tamponado para posterior processamento histopatológico.

Após a fixação, os fragmentos foram desidratados em etanol, diafanizados em xilol e incluídos em parafina. Posteriormente foram feitos cortes de $6 \mu \mathrm{m}$ de espessura em micrômetro de rotação, os quais foram corados pela hematoxilina e eosina.

Os resultados obtidos nas avaliações das provas de tuberculina, histopatológica e anatomopatológica foram dispostos em gráfico, tabelas de contingência e comparados através do cálculo de medidas de sensibilidade e especificidade diagnóstica.

\section{Resultados e discussão}

Os resultados da análise histopatológica diante do comportamento positivo ou suspeito dos bovinos à prova da tuberculinização e das lesões apresentadas ou não ao exame anatomopatológico estão discriminados nas Tabelas 1 e 2 , respectivamente.

Tabela 1: Freqüência dos resultados do exame histopatológico de bovinos, segundo o tipo de reação à tuberculinização

\begin{tabular}{lcccc}
\hline & & \multicolumn{3}{c}{ Histopatológico } \\
\cline { 3 - 5 } & & Positivo & Negativo & Total \\
\hline \multirow{3}{*}{ Tuberculinização } & Positiva & 6 & 28 & 34 \\
& Suspeita & 0 & 16 & 16 \\
& Total & 6 & 44 & 50 \\
\hline
\end{tabular}

Tabela 2: Freqüência dos resultados do exame histopatológico de bovinos positivos ou suspeitos à tuberculinização, segundo a sua condição anatomopatológica

\begin{tabular}{llcccc}
\hline & & \multicolumn{3}{c}{ Histopatológico } \\
\cline { 3 - 5 } & & & Positivo & Negativo & Total \\
\hline \multirow{4}{*}{ Anatomopatológico } & Positivo & Sugicostivo & 6 & 6 & 12 \\
& & 0 & 19 & 19 \\
& Negativo & & 6 & 25 & 31 \\
& Total & & 0 & 19 & 19 \\
& & 6 & 44 & 50 \\
\hline
\end{tabular}

Frente ao exame anatomopatológico, o histopatológico obteve uma baixa sensibilidade de $19 \%$, mas sua especificidade foi de $100 \%$, indicando ser um apreciável recurso auxiliar de diagnóstico da tuberculose em situações especiais, como a de abate de bovinos tuberculina positivos e suspeitos.

Comportamento semelhante tem sido observado em suínos. A presença de lesões macroscópicas tuberculóides em suínos, causadas por micobactérias atípicas e sem evidência de tuberculose à histopatologia, pode ser explicada, segundo Balian et al. (1997), de duas formas: o tipo de lesão desenvolvido pode se apresentar na forma de abscesso ou granuloma sem a presença de células epitelióides e células gigantes multinucleadas; os animais teriam sido abatidos antes de completar o período necessário ao aparecimento das referidas células, que ocorre aproximadamente aos 115 dias de infecção.

Mesmo assim, o diagnóstico da tuberculose bovina não deve prescindir do exame histopatológico, segundo Corner et al. (1990) e Reis et al. (1996).

A freqüência de animais tuberculina positivos com lesões anatomopatológicas (44\%) se aproximou da freqüência obtida por Corner et al. (1990), 52\%, quando empregaram a necrópsia detalhada. Esta proporção aumenta significativamente quando se emprega um exame anatomopatológico detalhado, ao invés da rotina de inspeção, geralmente devido ao aumento no diagnóstico de lesões únicas, que representam, segundo os mesmos, cerca de $58 \%$ das formas de tuberculose bovina. A sensibilidade do exame anatomo-patológico detalhado frente aos exames laboratoriais tem sido de cerca de $85 \%$, comportando-se como um satisfatório teste em inspeção post mortem (Corner et al., 1990).

A presença de micobactérias atípicas, como o M. intracelullare, em linfonodos mediastínicos e mesentéricos bovinos aparentemente sem lesões, foi atribuída, por Castro e Nemoto (1972), à relativa ausência de necrose de caseificação, que é comum na presença desta micobactéria.

As Tabelas 3 e 4 mostram, respectivamente, as distribuições das freqüências de bovinos e das lesões anatomopatológicas por tipo e comportamento à prova de tuberculinização.
Quanto ao desempenho do exame histopatológico, apenas $18 \%$ dos bovinos tuberculina positivos e nenhum dos suspeitos apresentaram lesões histológicas, sendo todas estas associadas com lesões anatomopatológicas típicas, 50\% calcificadas e $50 \%$ caseosas: e ainda, uma das lesões calcificadas (17\%) não revelou células gigantes multinucleadas, embora manifestasse as demais características histológicas de uma reação granulomatosa.
Tabela 3: Freqüência de bovinos com lesões anatomopatológicas (AP) segundo sua reação à tuberculinização (TB)

\begin{tabular}{|c|c|c|c|c|c|c|c|}
\hline \multirow{3}{*}{ TB } & \multicolumn{5}{|c|}{ AP com lesões } & \multirow{3}{*}{$\begin{array}{c}\mathrm{AP} \\
\text { sem lesões }\end{array}$} & \multirow{3}{*}{$\begin{array}{l}\mathrm{AP} \\
\text { total }\end{array}$} \\
\hline & \multicolumn{3}{|c|}{ típicas* } & \multirow[t]{2}{*}{ sugestivas } & \multirow[t]{2}{*}{ subtotal } & & \\
\hline & C & $c$ & Couc & & & & \\
\hline Positiva & 5 & 3 & 7 & 12 & 15 & 19 & 34 \\
\hline Suspeita & 0 & 1 & 1 & 5 & 6 & 10 & 16 \\
\hline Total & 5 & 4 & 8 & 17 & 21 & 29 & 50 \\
\hline
\end{tabular}

${ }^{*} \mathrm{C}=$ caseosas; $\mathrm{c}=$ calcificadas 
Tabela 4: Freqüência das lesões anatomopatológicas (AP) segundo sua reação à tuberculinização

\begin{tabular}{|c|c|c|c|c|c|c|}
\hline \multirow{3}{*}{ Tuberculinização } & \multicolumn{4}{|c|}{ AP com lesões } & \multirow{3}{*}{$\begin{array}{c}\mathrm{AP} \\
\text { sem lesões }\end{array}$} & \multirow{3}{*}{$\begin{array}{l}\mathrm{AP} \\
\text { tota }\end{array}$} \\
\hline & \multicolumn{2}{|c|}{ típicas* } & \multirow[t]{2}{*}{ sugestivas } & \multirow[t]{2}{*}{ subtotal } & & \\
\hline & $C$ & $c$ & & & & \\
\hline Positiva & 6 & 4 & 13 & 23 & 70 & 93 \\
\hline Suspeita & 0 & 2 & 6 & 8 & 46 & 54 \\
\hline Total & 6 & 6 & 19 & 31 & 116 & 147 \\
\hline
\end{tabular}

${ }^{*} \mathrm{C}=$ caseosas; $\mathrm{c}=$ calcificadas

O número de lesões encontradas por bovino tuberculina positivo foi de 1,53, próximo aos de Corner et al. (1990) em dois sistemas diferentes de abate $(1,54$ e 1,86). Para os bovinos suspeitos à tuberculinização, esta proporção decresceu para 1,33 lesões/animal.

Os órgãos, acompanhados das respectivas freqüências de lesões macroscó-picas detectadas, estão relacionados na Tabela 5.

Tabela 5: Freqüência das lesões anatomopatológicas detectadas por órgãos

\begin{tabular}{cccccc}
\hline Órgãos & \multicolumn{5}{c}{ Lesões } \\
\cline { 2 - 6 } & Caseosas & $\begin{array}{c}\text { Típicas } \\
\text { Calcificadas }\end{array}$ & Subtotal & Sugestivas & Total \\
& 0 & 0 & 0 & 10 & 10 \\
\hline Pré-escapular* $^{*}$ & 0 & 2 & 4 & 1 & 5 \\
Mediastínico-Bronquial* $_{\text {Hepático* }}^{*}$ & 0 & 0 & 0 & 2 & 2 \\
Mesentérico* & 1 & 1 & 2 & 0 & 2 \\
Ilíaco* & 0 & 0 & 0 & 3 & 3 \\
Retromamário* $_{\text {Pulmão }}$ & 0 & 0 & 0 & 1 & 1 \\
Fígado & 3 & 1 & 4 & 2 & 6 \\
Baço & 0 & 1 & 1 & 0 & 1 \\
Total & 0 & 1 & 1 & 0 & 1 \\
& 6 & 6 & 12 & 19 & 31 \\
\hline
\end{tabular}

*Linfonodos

Observa-se, na Tabela 5, que os locais eletivos para o diagnóstico da tuberculose bovina foram os tecidos respiratórios e digestivos, sobretudo os linfonodos medistínicos e bronquiais e o pulmão, coincidindo com os locais tidos como confiáveis por Corner et al. (1990), os quais consideram que $83,4 \%$ dos locais de lesões são representados pelo pulmão e pelos linfonodos mediastínico, bronquial e retrofaríngeo medial; representaram ainda, outros $11,5 \%$, o fígado e os linfonodos mesentérico, hepático, mandibular e parotidiano.

Segundo Corner et al. (1990), as lesões de tuberculose são encontradas mais freqüentemente em órgãos ricos em tecidos reticuloendoteliais, particularmente pulmão, linfonodos e fígado. Segundo os mesmos autores, Lepper e Pearson (1973) encontraram $70-90 \%$ das lesões nos pulmões e linfonodos da cabeça e cavidade torácica, mas enfatizaram que as lesões podem ser encontradas em quaisquer linfonodos.

A presença de elevado número de lesões em linfonodos cervical superficial (quarto dianteiro) e ilíaco (traseiro), alerta para os riscos de contaminação da carcaça. Todavia, este resultado pode não refletir a distribuição real de lesões no organismo bovino, pois foram os locais privilegiados na amostragem desta pesquisa.

As lesões consideradas típicas (24\%) foram diagnosticadas na superfície de incisão dos órgãos e se apresentaram basicamente em três formas: caseosas $(33 \%)$, calcificadas $(50 \%)$ ou purulentas $(17 \%)$.

As lesões caseosas geralmente se representaram por nódulos amarelados, de consistência mole, esféricos, únicos ou múltiplos, com diâmetro variável, predominando entre 1,5 e $2,5 \mathrm{~cm}$. Entre os nódulos calcificados, predominaram os de tamanho pequeno, até $1 \mathrm{~cm}$ de diâmetro; duas lesões calcificadas, uma no baço (única) e outra no fígado (múltipla) se destacaram pela sua intensa reação calcárea, com formação de grânulos endurecidos, maiores, perceptíveis ao corte (ranger). As lesões denominadas purulentas se referiram a evidentes reações inflamatórias agudas ou crônicas, disseminadas, com secreções não tipicamente caseosas, com ou sem aderências.

As lesões consideradas sugestivas de tuberculose em bovinos positivos ou suspeitos à tuberculinização (38\%) foram agrupadas entre as lesões encontradas em órgãos mais susceptíveis à tuberculose, com características nodulares ou não, predominantemente hemorrágicas, de tamanhos e formas variados. Nestes órgãos também foram encontradas lesões inespecíficas que foram incluídas no grupo das ausentes (38\%).

Conforme se observa na Figura 1, predominaram na amostragem estudada, os perfis de diagnóstico voltados para resultado histopatológico negativo na ausência de lesões macroscópicas ou na presença de lesões apenas sugestivas, tanto nos bovinos tuberculina positivos, quanto nos suspeitos.

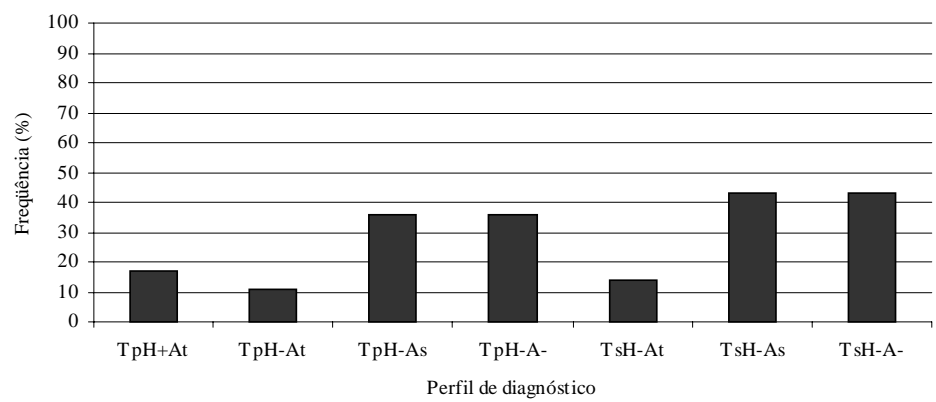

Figura 1: Freqüência dos perfis de diagnóstico da tuberculose bovina: tuberculinização positiva (Tp) ou suspeita (Ts), exame histopatológico positivo $(\mathrm{H}+)$ ou negativo $(\mathrm{H}-)$ e exame anatomopatológico típico (At), sugestivo (As) ou negativo (A-).

É importante considerar a possibilidade de ocorrerem lesões anatomopatológicas em bovinos e suínos abatidos sem revelar a presença de micobactérias (Balian et al., 1997). Esta circunstância também reforça a indicação do emprego de 
outros métodos auxiliares de diagnóstico, de preferência mais específicos que o exame anatomopatológico, para aumentar a eficácia do julgamento sanitário na inspeção da carne, evitando ainda o rigor exagerado nas condenações.

Analisando um grande número de amostras de bovinos com lesões anatomopatológicas atribuídas a princípio à tuberculose, Tammemagi et al. (1973) verificaram, por intermédio de exame histopatológico, que 10,5\% destas lesões eram sugestivas de actinobacilose e 5,3\% correspondiam a outras lesões incompatíveis com tuberculose. Norton (1976) também associou o aparecimento de lesões macroscópicas sugestivas de tuberculose a outros agentes, isolando microrganismos pertencentes ao grupo Nocardia/Streptomyces. Foi isolada a bactéria Nocardia asteroides em tecido muscular bovino com reações granulomatosas, que se confundem macro e microscopicamente com as lesões de tuberculose (Benites et al., 1999).

\section{Conclusões}

As freqüências de bovinos com lesões anatomopatológicas e de lesões por animal foram maiores quando os animais eram tuberculina positivos, em comparação aos suspeitos.

Apenas $18 \%$ dos bovinos tuberculina positivos e nenhum dos suspeitos apresentaram lesões histológicas, sendo todas associadas com lesões anatomopatológicas típicas, 50\% calcificadas e $50 \%$ caseosas.

\section{Referências}

ANTUNES, J. L. F.; WALDMAN, E. A. A tuberculose através do século: séries temporais para a mortalidade em São Paulo, Brasil, 1900-97. Cad. Saúde Pública, Rio de Janeiro, v. 15, n. 3, 1999.

BALIAN, S. C.; RIBEIRO, P.; VASCONCELLOS, S. A.; PINHEIRO, S. R.; FERREIRA-NETO, J. S., GUERRA, J. L.; XAVIER, J. G.; MORAIS, Z. M.; TELLES, M. A. S. Linfadenites tuberculóides em suínos abatidos no Estado de São Paulo, Brasil: aspectos macroscópicos, histopatológicos e pesquisa de micobactérias. Rev. Saúde Pública, v. 31, n. 4, p. 391397, 1997.

BENITES, N. R.; BALIAN, S. C.; FERREIRANETO, J. S.; SAKAMOTO, S. M.; GUERRA, J. L.; SOUZA, S. L. P.; PANETTA, J. C. Isolamento de Nocardia asteroides de lesão granulomatosa em carne bovina. Higiene Alimentar, v. 13, n. 59, p. 35-38, 1999.

BRASIL. Leis, decretos, etc. Regulamento de inspeção industrial e sanitária de produtos de origem animal. Aprovado pelo decreto ํo 30.691 de 29/3/52, alterado pelo decreto oㅜ1.255, de 25/6/1962. Brasília, DF, Ministério da Agricultura, 1980

BRASIL/MA. Inspeção de carnes; padronização de técnicas, instalações e equipamentos. Brasília, DF, DIPOA/DICAR, 1971.

CASTRO, A. F. P.; NEMOTO, H. Occurrence of atypical mycobacteria in the lymphonodes of apparently healthy slaughtered cattle in São PauIo, Brasil. Rev. microbiol., v. 3, n. 2, p. 75-78, 1972.

CENTRO Panamericano de Zoonosis - CPZ. Manual de normas y procedimientos técnicos para la bacteriología de la tuberculosis III. Sensibilidad del Mycobacterium tuberculosis a las drogas. La identificación de micobacterias. Buenos Aires, 1986. (Nota técnica, 28). CORNER, L. A.; MELVILLE, L.; McCUBBIN, K.; SMALL, K. J.;McCORMICK, B. S.; WOOD, P. R.; ROTHEL, J. S. Efficiency of inspection procedures
Os locais destacados no diagnóstico da tuberculose bovina foram os tecidos respiratórios e digestivos, sobretudo os linfonodos mediastínicos e bronquiais e o pulmão.

Predominaram os perfis de diagnóstico correspondentes aos resultados histopatológico negativo, na ausência de lesões macroscópicas ou na presença de lesões apenas sugestivas, tanto nos bovinos tuberculina positivos, quanto nos suspeitos.

O exame histopatológico apresenta menor sensibilidade, entretanto, maior especificidade que o anatomopatológico no diagnóstico de lesões de tuberculose em tecidos bovinos. Porém, quando o exame anatomopatológico é realizado com método detalhado, este se apresenta como um teste mais sensível, comportando-se como um satisfatório exame em inspeção post mortem.

Os programas de tuberculinização dos rebanhos e de inspeção de sua carne têm sido importantes recursos de rastreabilidade da tuberculose detectada em matadouros, resultando em redução dos números de animais infectados. Entretanto, os benefícios do controle da tuberculose bovina para a saúde pública e animal dependem de novas técnicas e procedimentos laboratoriais, como o exame histopatológico, bem como do aprimoramento do exame anatomopatológico rotineiramente empregado na inspeção post mortem, sobretudo em casos de bovinos tuberculina positivos ou suspeitos.

for the detection of tuberculous lesions in catle. Australian Veterinary Journal, v. 67, n. 11, p. 389-392, 1990.

GRANGE, J. M.; YATES, M. D. Zoonotic aspects of Mycobacterium bovis infection. Vet. microbiol., v. 40, p. 137-151, 1994.

LANGENEGGER, C. H.; LANGENEGGER, J.; RAMOS, A. A. Reações alérgicas inespecíficas no diagnóstico da tuberculose em bovinos causadas por Mycobacterium intracellulare. Pesq. Agropec. Bras., Ser. Vet., v. 11, p. 65-71, 1976.

NELSON, A. M. The cost of disease eradication. Smallpox and bovine tuberculosis. Ann. N. Y. Acad. Sci., v. 894, p. 83-91, 1999.

NORTON, J. H. Tuberculosis-like granulomas in cattle caused by Actinomycetes. Aust. Vet. J., v. 52, p. 455-457, 1976.

REIS, D. O.; ALMEIDA, L. P.; FARIA, A. R. Comparative study of the macroscopic changes in the lymph nodes of slaughtered cattle with lymphosarcoma, tuberculosis and lymphadenitis. Higiene Alimentar; v. 9, p. 28-30, 1995.

REIS, D. O.; COELHO, H. E.; LUCIO, N. F. Morphological characteristics of tuberculosis lesions in bovine organs and lymph nodes. Higiene Alimentar, v. 10, n. 41, p. 15-16, 1996.

ROBERTS, T. A retrospectives assessment of human health protection benefits from removal of tuberculous beef. J. Fd. Prot., v. 49, n. 4, p. 293-298, 1986.

SOUZA, A. V.; SOUZA, C. F. A.; SOUZA, R. M.; RIBEIRO, R. M. P.; OLIVEIRA, A. L. A importância da tuberculose bovina como zoonose. Higiene Alimentar, v. 13, n. 59, p. 22-27, 1999.

TAMMEMAGI, L.; SIMMONS, G. C.; KELMAN, R.; HALL, W. T. K. A study of tuberculosis-like lesions in cattle slaughtered in Queensland meatworks. Aust. Vet. J., v. 49, p. 507-511, 1973. 